\title{
Clinical effect of Resina Draconis capsules on primary dysmenorrhoea
}

\author{
Li Sun* and Jia Wang \\ Department of Gynecology, Binzhou Medical University Hospital, Binzhou, Shandong, 256603, PR China \\ ${ }^{*}$ For correspondence: Email: sunlisltjpr@163.com
}

Received: 5 May 2016

Revised accepted: 15 August 2016

\begin{abstract}
Purpose: To examine the effectiveness of Resina Draconis capsules in the treatment of primary dysmenorrhoea.

Methods: In total, 324 patients with primary dysmenorrhoea were randomly allocated to three groups based on treatment with capsules containing Resina Draconis, Leonurus japonicus Houtt., or a placebo for 3 months. The patients' visual analogue scale (VAS) scores and dysmenorrhoea symptoms were evaluated.

Results: VAS scores of the Resina Draconis, L. japonicus, and placebo groups decreased from $7.31 \pm$ $1.36,7.12 \pm 1.65$, and $7.25 \pm 1.47$ to $3.35 \pm 1.43,5.27 \pm 1.24$, and $7.08 \pm 2.10$, respectively. The change was greatest for the Resina Draconis group $(p<0.01)$. The incidence of symptoms associated with dysmenorrhoea decreased in all three groups, but the change was greatest for Resina Draconis group $(p<0.01)$. Overall, Resina Draconis was more effective than L. japonicus $(94.40$ vs. $72.20 \%)(p$ $<0.05)$.

Conclusion: Resina Draconis capsules are effective in relieving primary dysmenorrhoea and lowering the incidence of symptoms associated with dysmenorrhoea.
\end{abstract}

Keywords: Primary dysmenorrhoea, Resina Draconis, Leonurus japonicus Houtt., Visual analogue scale

Tropical Journal of Pharmaceutical Research is indexed by Science Citation Index (SciSearch), Scopus, International Pharmaceutical Abstract, Chemical Abstracts, Embase, Index Copernicus, EBSCO, African Index Medicus, JournalSeek, Journal Citation Reports/Science Edition, Directory of Open Access Journals (DOAJ), African Journal Online, Bioline International, Open-J-Gate and Pharmacy Abstracts

\section{INTRODUCTION}

Dysmenorrhoea is a common gynaecological disease that can be classified into primary and secondary dysmenorrhoea. Primary dysmenorrhoea, or functional dysmenorrhoea, refers to pain, bloating, and discomfort in the lower abdomen before and after menstruation or during menstruation when there is no organic disease in the reproductive organs. In severe cases, it can even lead to nausea, emesis, cold hands and feet, and syncope. The estimated incidence of primary dysmenorrhoea in China is $36 \%[1,2]$. The occurrence of primary dysmenorrhoea is correlated with prostaglandin released by the endometrium during menstruation; the prostaglandin levels in the endometrium and menstrual blood of women with dysmenorrhoea are higher than in healthy women [3]. Primary dysmenorrhoea usually occurs $6-12$ months after the first menstrual cycle [4].

The pathogenesis of primary dysmenorrhoea is complex and includes mental and neural factors. It can also be induced by uterine ischemia caused by the spasmodic contraction of uterine muscle. Uterine contractions stimulated by the retention of menstrual blood resulting from uterine dysgenesis, a narrowed cervix, or uterine 
hyperflexion, may also lead to primary dysmenorrhoea [5]. The incidence of primary dysmenorrhoea has increased in recent years, impacting the quality of life for affected women [6]. Primary dysmenorrhoea usually disappears after a delivery.

The main components of Resina Draconis capsules include Resina Draconis, pseudoginseng, and borneol [7]. In traditional Chinese medicine, Qi controls the blood and blood is the mother of Qi; stasis is eliminated when blood circulates, and blood circulates when $\mathrm{Q} i$ is active. Resina Draconis, a red resin produced by plants of the genus Dracaena Vand. ex L, has a long history of use in China. It is believed to function in activating blood, eliminating stasis, relieving swelling and pain, and stopping bleeding [8]. Resina Draconis capsules contain several active ingredients, including Resina Draconis, saponins, and plant defensins, which have double regulatory effects. Although there are many treatments for primary dysmenorrhoea, few studies have examined treatment with Resina Draconis. This study examined the use and clinical effects of Resina Draconis capsules in the treatment of primary dysmenorrhoea.

\section{EXPERIMENTAL}

\section{Study setting and patient characteristics}

In total, 324 patients with confirmed primary dysmenorrhoea in Binzhou Medical University Hospital, Shandong, China, were enrolled in this study, which was approved by the medical ethics committee of Binzhou Medical University Hospital (approval no. BMUHSL20150425) and conducted according to the tenets of the Declaration of Helsinki [9].

The enrolled patients had no organic lesions in their reproductive organs, based on B-mode ultrasonography, had menstrual cycles of $23-35$ days, and provided written informed consent. Patients were excluded if they were pregnant or breastfeeding, if they had a mental illness or a major disease related to the heart, liver, kidney, or haemopoietic systems, or if they were allergic to the drugs. The enrolled patients were allocated randomly to Resina Draconis, Leonurus japonicus Houtt., and placebo treatment groups ( $n=108$ per group).

Subjects in the Resina Draconis group had an average age of $28 \pm 5.70$ (range $15-35$ ) years and disease duration of $3.40 \pm 2$ (range $2-6$ ) years, 78 were single and 30 were married, 60 patients had previously been pregnant, and 18,
60 , and 30 patients had mild, moderate, and severe dysmenorrhoea, respectively. In the $L$. japonicus group, the average age was $28.10 \pm$ 6.30 (range $14-32$ ) years and the disease duration was $4 \pm 2.40$ (range $1-6$ ) years, 66 patients were single and 42 were married; 66 patients had previously been pregnant, and 30 , 60 and 18 patients had mild, moderate, and severe dysmenorrhoea, respectively. In the placebo group, the average patient age was $29.30 \pm 6.70$ (range $13-36$ ) years and the average disease duration was $3.60 \pm 2.20$ (range $2-5$ ) years; 69 patients were single and 39 were married, 63 patients had previously been pregnant, and 24, 72 and 12 patients had mild, moderate, and severe dysmenorrhoea, respectively. None of the differences among the groups was significant.

\section{Treatment}

Each patient in the Resina Draconis group was given two Resina Draconis capsules (Yunhe Pharmaceutical, Yunnan, China; main component: Resina Draconis, auxiliary components: starch, sodium carboxymethyl starch, and lactose) three times daily. Each member of the $L$. japonicus group was given one L. japonicus Houtt. capsule (Shenyang Yongda Pharmaceutical, Liaoning, China; main component: $L$. japonicus Houtt.) three times daily. Each patient in the placebo group was given a single placebo capsule (Kangyuan Pharmaceutical, Jiangsu, China; main components: lactose and a pigment), three times daily. The capsules were taken orally for 1 week each month, beginning 3 days before the anticipated menstrual period, for 3 months.

\section{Observation index}

The patients used a visual analogue scale (VAS) to score their pain before and after treatment. The scale ranged from 0 to 10 points, with 0 points indicating no pain and 10 points intolerable pain. The relief of dysmenorrhoea symptoms (e.g., lower abdominal pain and distension, lower abdominal crymodynia, premenstrual breast swelling, cold limbs, and nausea and vomiting), the presence of blood in urine, stool indexes; and liver and kidney functions were observed in all three groups. After treatment, all patients were followed for 3 months. The curative effect was compared among the three groups.

\section{Evaluation criteria for a cure}

Cure was defined as the disappearance of abdominal pain and other symptoms, and no 
recurrence of disease after drug withdrawal. The result was marked if the patients had significantly reduced abdominal pain, if the other clinical symptoms disappeared or were reduced, and if they did not need to take analgesics. The treatment was considered effective if the patients experienced relief from symptoms associated with dysmenorrhoea and stopped taking analgesics. Treatment was considered ineffective if there were no changes in their symptoms and the patients still required analgesics.

\section{Statistical analysis}

The data were analysed using the SPSS software (ver. 15.0) and compared using t-tests, one-way analysis of variance, and $q$ tests. Enumerated data were assessed using the $x^{2}$ test. Ordered categorical data were tested with the rank sum test. Differences were considered to have statistical significance if $p<0.05$.

\section{RESULTS}

\section{Comparison of VAS scores before and after treatment}

Before treatment, the differences in VAS scores among the three groups were not significant $(p>$ 0.05). The VAS scores before and after treatment did not differ in the placebo group $(p>$ 0.05). After treatment, the VAS scores of the Resina Draconis and L. japonicus groups were lower than that of the placebo group, and they were much lower for the Resina Draconis group $(p<0.05)$ (Table 1).

\section{Comparison of associated symptoms before and after treatment}

Before treatment, the incidence of symptoms associated with dysmenorrhoea, including abdominal pain and distension, abdominal crymodynia, premenstrual breast swelling, cold limbs, and nausea and vomiting, did not differ significantly among the three groups. After treatment, such symptoms were less frequent in the Resina Draconis and $L$. japonicus groups compared with the placebo group $(p<0.01)$. In addition, abdominal pain and distension, abdominal crymodynia, and nausea and vomiting were less frequent in the Resina Draconis group compared with the $L$. japonicus group after treatment $(p<0.05$ or 0.01$)$. The incidence of symptoms associated with dysmenorrhoea in the placebo group did not differ before and after treatment $(p>0.05)$ (Table 2$)$.

\section{Comparison of clinical effect among the three groups}

At the 3-month follow-up visit, the curative effect differed significantly among the three groups ( $\mathrm{Hc}$ $=58.461, p<0.01$ ); it was $94.4 \%$ in the Resina Draconis group versus $72.2 \%$ in the $L$. japonicus group $(p<0.05)$ (Table 3$)$.

Table 1: Comparison of the VAS scores before and after treatment (mean \pm standard deviation [SD])

\begin{tabular}{lccc}
\hline Group & $\mathbf{N}$ & Before & After \\
\hline Resina Draconis & 108 & $7.31 \pm 1.36$ & $3.35 \pm 1.43^{\mathrm{abc}}$ \\
Leonurus japonicus & 108 & $7.12 \pm 1.65$ & $5.27 \pm 1.24^{\mathrm{ab}}$ \\
Placebo & 108 & $7.25 \pm 1.47$ & $7.08 \pm 2.10$ \\
\hline${ }^{\mathrm{a} P<0.01 \text { for within-group comparisons, }}{ }^{\mathrm{b}} p<0.01$ compared with the placebo group \\
after treatment, ${ }^{c} p<0.01$ compared with the $L$. japonicus group after treatment
\end{tabular}

Table 2: Comparison of symptoms associated with dysmenorrhoea before and after treatment (N, \%)

\begin{tabular}{lcccccc}
\hline \multirow{2}{*}{ Symptom } & \multicolumn{2}{c}{ Resina draconis } & \multicolumn{2}{c}{ L. japonicus } & \multicolumn{2}{c}{ Placebo } \\
& Before & After & Before & After & \multicolumn{1}{c}{ Before } & After \\
\hline Abdominal pain and distension & $99(91.70)$ & $10(13.90)^{\mathrm{abd}}$ & $105(97.20)$ & $45(41.70)^{\mathrm{ab}}$ & $102(94.40)$ & $90(83.30)$ \\
Abdominal crymodynia & $87(89.60)$ & $6(8.30)^{\mathrm{abc}}$ & $84(77.80)$ & $30(27.80)^{\mathrm{ab}}$ & $90(83.30)$ & $72(66.70)$ \\
Premenstrual breast swelling & $84(77.80)$ & $10(13.90)^{\mathrm{ab}}$ & $81(75)$ & $27(25)^{\mathrm{ab}}$ & $87(89.60)$ & $84(77.80)$ \\
Cold limbs $_{\text {Nausea and vomiting }}$ & $96(88.90)$ & $10(13.90)^{\mathrm{ab}}$ & $99(91.70)$ & $36(33.30)^{\mathrm{ab}}$ & $87(89.60)$ & $81(75)$ \\
${ }^{\mathrm{a}} P<0.01$ before and after treatment in the different groups, ${ }^{\mathrm{b}} p<0.01$ compared with the placebo group after \\
treatment, ${ }^{c} p<0.05,{ }^{d} p<0.01$ compared with the $L$. japonicus group after treatment
\end{tabular}

Table 3: Comparison of the curative effect in the three groups $(\mathrm{N}, \%)$

\begin{tabular}{lccccc}
\hline Group & N & Cure & Marked effect & Effective & Ineffective \\
\hline Resina Draconis & 108 & $30(27.80)$ & $54(50)$ & $18(16.70)$ & $6(5.50)$ \\
L. japonicus & 108 & $27(25)$ & $39(36.10)$ & $12(11.10)$ & $30(27.80)$ \\
Placebo & 108 & 0 & 0 & 0 & $108(100)$ \\
\hline
\end{tabular}




\section{Safety of drug use}

The patients in the three groups developed no obvious untoward reactions during treatment. In addition, blood, urine, and stool indexes and the renal and kidney functions of the patients in the three groups were normal before and after treatment.

\section{DISCUSSION}

Primary dysmenorrhoea, or functional dysmenorrhoea, occurs mostly in menarche and in nulliparous women. Patients with the disease have no obvious pathological changes to the reproductive organs on examination. The pathogenesis of primary dysmenorrhoea is unclear. Pathogenic factors contributing to the disease include uterine contraction abnormalities, cyclic changes in oestrogens and androgens in the menstrual cycle, and ischaemia and the stimulation of the nerve plexus of the uterine isthmus [10].

During menstruation, the endometrium synthesises and releases more prostaglandins, which can lead to spasmodic uterine contractions and cause pain. Excessive uterine contractions are also correlated with oxytocin levels. About $10 \%$ of women have primary dysmenorrhoea during their lifetime, and the incidence of primary dysmenorrhoea is increasing [11]. The treatment of primary dysmenorrhoea includes non-steroidal anti-inflammatory drugs to relieve the pain and spasms [12]. Although such drugs work rapidly, their side effects include gastrointestinal symptoms and a heavier menstrual flow, which can reduce acceptance of the drugs by patients [13].

In traditional Chinese medicine, the occurrence of dysmenorrhoea is associated with various factors [14-16], including catching a cold during the menstrual period, eating cold food, and sitting on wet ground. It can also be induced by liver Qi stagnation, weakness of the spleen and stomach, a congenital deficiency in Qi and the blood, as well as deficiencies of the liver and kidneys. The main symptoms of dysmenorrhoea of the congealing cold and blood stasis types are lower abdominal crymodynia, reduced blood volume, dark coloured blood, blood clots, cold limbs, a light or dark purple tongue, and a white furred tongue during the menstrual period. Catching a cold during the menstrual period or eating cold food can induce the invasion of cold pathogens and cold congealing in the uterus, and consequently block the circulation of $\mathrm{Qi}$ and blood.
Resina draconis capsules can relieve or reduce the pain of patients with primary dysmenorrhoea, shorten the duration of dysmenorrhoea, and improve the syndromes of traditional Chinese medicine $[17,18]$. Leonurus japonicus can relieve the pain caused by dysmenorrhoea by restraining the activity of a spasmodic uterus, lowering the prostaglandin levels in smooth muscle, and increasing progesterone levels. The compounds loureirin and saponin in Resina draconis not only improve microcirculation, regulate metabolism, and enhance immune function, but also activate blood, eliminate blood stasis, and stop bleeding [19].

This study showed that, both Resina draconis and $L$. japonicus reduced the pain of patients with dysmenorrhoea, and Resina draconis was significantly better $(p<0.01)$. The incidences of abdominal pain and distension, lower abdominal crymodynia, and nausea and vomiting were lower in the Resina Draconis group than in the $L$. japonicus group $(p<0.05$ or 0.01 ).

\section{Study limitations}

The sample size was relatively small due to limited time and the experimental conditions. In addition, the effects of diet and emotion on the outcome were not completely excluded due to patient compliance.

\section{CONCLUSION}

Resina draconis capsules effectively relieved the pain associated with primary dysmenorrhoea and reduced the incidence of symptoms associated with dysmenorrhoea. Thus, Resina Draconis may potentially be useful in the clinical management of dysmenorrhoea, but further studies, including toxicological investigations, are required.

\section{DECLARATIONS}

\section{Acknowledgement}

The authors sincerely thank all who supported this work.

\section{Conflict of Interest}

No conflict of interest associated with this work.

\section{Contribution of Authors}

The authors declare that this work was done by the authors named in this article and all liabilities pertaining to claims relating to the content of this article will be borne by them. 


\section{REFERENCES}

1. Tian $\mathrm{QH}, \mathrm{Hu} Y \mathrm{YH}$, Jiao SY. Observation of tongjingshu decoction combined with conventional acupuncture treatment of Dong's extra-point on dysmenorrheal. Hebei J Trad Chin Med 2014; 36(4): 504-506.

2. Chan SS C, Yiu KW, Yuen PM, Sahota DS, Chung TRH. Menstrual problems and health-seeking behaviour in Hong Kong Chinese girls. Hong Kong Med J 2009; 15(1): 18-23.

3. Wang Q. Clinical observation of angelica sini decoction in the treatment of code type dysmenorrhea. Chin Med Mod Dist Edu China 2014; 12(15): 33-34.

4. Proctor ML, Murphy PA, Pattison HM, Suckling J, Farquhar CM. Behavioural interventions for primary and secondary dysmenorrhea. Cochrane Database Syst Rev 2007; (3): D2248.

5. Chou MH, Wang HH. Predictors of dysmenorrhea and self-care behavior among vocational nursing school female students. J Nurs Res 2008; 16(1): 17-25.

6. Zhu XP, Smith $C$, Bensoussan A. Chinese herbal medicine for primary dysmenorrhoea. Cochrane Database Syst Rev 2007; 4(2): CD005288.

7. Liang WY, Zhang XZ, Bai WP, Yu XM, Wang AQ. Clinical observation of 105 patients with motherwort compound in the treatment of primary dysmenorrhea. Beijing J Trad Chin Med 2006; 25(8): 510-511.

8. Xiang JL, Cheng R. Research of the activating blood and hemostasis effect of dragon's blood. W China J Pharm Sci 2000; 15(6): 430-434.

9. Declaration of Helsinki. The 59th World Medical Association, 2008.
10. Pu BC, Jiang GY, Fang L. Research on pain related factors and associations of primary dysmenorrhea. Chin Arch Trad Chin Med 2014; 32(6): 1368-1370.

11. Huang H. 32 cases of dymenorrhea treated with wenjing decoction. Pub Med Forum Mag 2010; 14(20): 622.

12. Dang HZ, Teng JX. An overview of Chinese and western medicine on primary dysmenorrhea. J Hunan Coll Trad Chin Med 2000; 20(3): 70-72.

13. $Y u X W$, Jin $H$, Han Z, Li F, Wang FQ, Zhang $Y J, W u Y L$. Analysis on affecting factors of primary dysmenorrhea in adolescent girls. Chin J Behav Med Sci 2003; 12(3): 273-275.

14. Cao YX. Pathogenesis and treatment of primary dysmenorrhea. Chin J Pract Gynecol Obstet 2001; 17(4): 205-207.

15. Yan CQ, Wang TL. 40 cases of primary dysmenorrhea treated with motherwort compound combined with fenbid. Nei Mongol J Trad Chin Med 2011; 30(12): 58.

16. Ji B, Ren XX, Zhao YF, Song XL, Xu LL, Zhang LF, Zhu $J$. Comments on study of pathogenesy, prevention and treatment of primary dysmenorrhea. China J Mod Med 2008; 18(13): 1856-1858.

17. Zeng LB, Zhang JP. Pharmacology and clinical application of traditional Chinese medicine-dragon's blood. Asia-Pacific Trad Med 2014; 10(3): 43-44.

18. Luo SC, Hu RX, Shi D. Basic research and clinical application of dragon's blood. Mod J Integr Chin Trad West Med 2001; 10(23): 2327-2329.

19. Yang $X Y$, Yao L. Pharmacological action and clinical experience of dragon's blood. Heilongjiang Med 2011; 24(2): $265-266$ 